\title{
Thermal, Structural, and Viscoelastic Characterization of cis-Poly(phenylene vinylene) Related to its Photo-Isomerization
}

\author{
Hiroyuki Katayama, Fumiyuki Ozawa, Yumi Matsumiya, and Hiroshi Watanabe ${ }^{\dagger}$ \\ Institute for Chemical Research, Kyoto University, Gokasho, Uji 611-0011, Japan
}

(Received August 24, 2005; Accepted October 17, 2005; Published February 15, 2006)

\begin{abstract}
A cis-poly(phenylene vinylene) (cis-PPV) recently synthesized by Katayama, Ozawa, and coworkers undergoes photo-isomerization into trans configuration. This isomerization occurred rather quickly within $45 \mathrm{~min}$ in solvent-cast films at room temperature. The cis-PPV chains exhibiting this quick isomerization were expected to be highly mobile in the bulk state, and this expectation was examined from thermal, structural, and viscoelastic tests for a cis-PPV sample with $M_{\mathrm{n}}=4300$ and $M_{\mathrm{w}} / M_{\mathrm{n}}=2.16$. It turned out that the as-polymerized cis-PPV was partially crystalline and fully melted at $T_{\mathrm{m}} \cong 109^{\circ} \mathrm{C}$. The samples quenched from a molten state to lower temperatures $\left(\leq 70^{\circ} \mathrm{C}<T_{\mathrm{m}}\right)$ as well as cast from a solution were amorphous for few hours, enabling the viscoelastic test in the fully amorphous state. The WLF-type time-temperature superposition was valid for these quenched and/or cast sample, confirming the amorphous character of the cis-PPV chains therein. The terminal viscoelastic relaxation time of these chains, equivalent to a time required for thermal changes in a large-scale conformation, was $\cong 10 \mathrm{~s}$ at room temperature. This result suggested that the conformational changes of the amorphous chains (the major component in the cast films) were sufficiently fast to allow the efficient cis-to-trans photo-isomerization. [DOI 10.1295/polymj.38.184]

KEY WORDS Poly(phenylene vinylene) / Semicrystalline / Viscoelastic Characterization /
\end{abstract}

Recently, Katayama, Ozawa, and coworkers conducted a stereo-controlled synthesis of poly[(2,5-dioctyloxy-1,4-phenylene vinylene)-co-(1,4-phenylene vinylene)]s (PPV) using the Hiyama- and SuzukiMiyaura-type polycondensation methods. ${ }^{1,2}$ The PPV, having the structure shown in Scheme 1, is a $\pi$-conjugated polymer offering applications to light-emitting diodes, ${ }^{3}$ solar cells, ${ }^{4}$ and so on. In these applications, it is very useful if we can make arbitrary patterning of thin PPV films cast on appropriate substrates. Katayama, et al. ${ }^{1}$ showed that the cis-to-trans photoisomerization by UV light occurred rather quickly within $45 \mathrm{~min}$ in the cis-PPV film prepared by solventcasting and that the resulting trans-PPV film, being partly crosslinked on the UV radiation, was insoluble in solvents while the cis-PPV film was soluble (in chloroform, for example). This photo-isomerization, followed by rinsing with solvents, provides us with an interesting route of pattern-writing in the residual
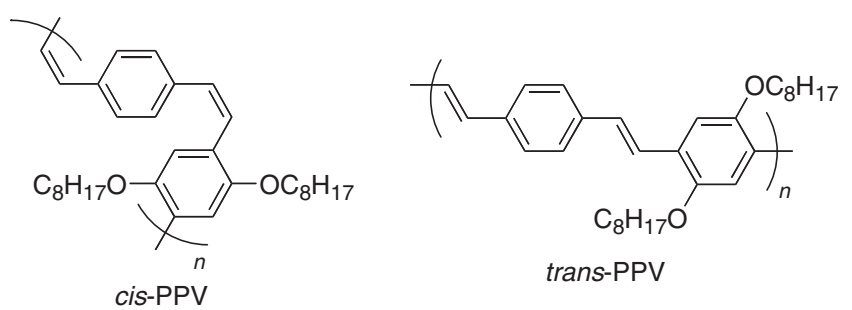

Scheme 1. Chemical Structure of cis- and trans-PPVs.
trans-PPV film by use of appropriate UV masks, as already demonstrated previously. ${ }^{1}$

Because the photo-isomerization occurs rather quickly, the cis-PPV chains in the solvent-cast film are expected to be essentially in the amorphous state thereby exhibiting fast changes of their large-scale conformation. (It is quite unlikely that the isomerization occurs quickly for the chains in a rigid crystalline state.) However, no details have been elucidated for the thermal motion of the amorphous cis-PPV chains. Thus, we have conducted thermal, structural, and viscoelastic tests for a previously synthesized cis-PPV sample to examine this motion. It turned out that the cis-PPV chains were indeed in the amorphous state for few hours after the heat-treatment (quenching) and/or solvent casting and that the thermal motion of those amorphous chains occurred quickly (within $\cong$ $10 \mathrm{~s}$ at room temperature), as expected. Details of these results are presented in this paper.

\section{EXPERIMENTAL}

\section{Material}

An all-cis poly(phenylene vinylene) (cis-PPV) sample synthesized in the previous work $^{1}$ was utilized. The apparent number-average molecular weight and the polydispersity index, both determined from GPC calibration with polystyrene standards, were $M_{\mathrm{n}}=$ 4300 and $M_{\mathrm{w}} / M_{\mathrm{n}}=2.16$, respectively. ${ }^{1}$ The as-poly-

${ }^{\dagger}$ To whom correspondence should be addressed (Tel: +81-774-38-3135, Fax: +81-774-38-3139, E-mail: hiroshi@ scl.kyoto-u.ac.jp). 
merized sample was obtained in a powder form, and the heat-treated and/or solvent cast sample was a sticky bulk material at room temperature.

\section{Measurements}

For the cis-PPV sample, differential scanning calorimetric (DSC) measurements were conducted with a calorimeter (Diamond DSC, Perkin Elmer Co.) equipped in Professor Yoko's laboratory in Institute for Chemical Research, Kyoto University. The as-polymerized sample was heated from -50 to $150^{\circ} \mathrm{C}$ at a heating rate of $20^{\circ} \mathrm{C} / \mathrm{min}$ and then quenched from 160 to $-60^{\circ} \mathrm{C}$. For this quenched sample, the second and third runs were made with the same protocol. The quenched sample after the third run was annealed at $25^{\circ} \mathrm{C}$ for $24 \mathrm{~h}$, and the fourth and fifth runs were made in the way similar to that for the first and second runs. For comparison, the DSC measurement was made also for trans-PPV sample recovered from a dilute benzene solution of cis-PPV chains irradiated with UV light $\left(2.1 \mathrm{~mW} \mathrm{~cm}^{-2}\right)$.

For the as-polymerized cis-PPV sample as well as the sample quenched from 160 to $25^{\circ} \mathrm{C}$ and annealed at $25^{\circ} \mathrm{C}$ for $24 \mathrm{~h}$, wide-angle X-ray diffraction (WAXD) measurements were conducted at $25^{\circ} \mathrm{C}$ with a laboratory goniometer (RINT-2000, Rigaku Co.) equipped in Professor Kanaya's laboratory in Institute for Chemical Research, Kyoto University. The $\mathrm{X}$-ray wavelength was $\lambda=0.154 \mathrm{~nm}\left(\mathrm{Cu}-\mathrm{K}_{\alpha}\right.$ line $)$. The WAXD intensity $I(\theta)$ was measured as a function of the scattering angle $2 \theta$, and no further correction (such as the desmearing correction) was made.

The DSC measurements revealed that the as-polymerized cis-PPV sample was partially crystalline (and fully melted at $T_{\mathrm{m}} \cong 109^{\circ} \mathrm{C}$ ) but the quenched sample remained in the supercooled amorphous state for few hours. Thus, for the sample quenched from $120^{\circ} \mathrm{C}\left(>T_{\mathrm{m}}\right)$ to several temperatures between 26 and $70^{\circ} \mathrm{C}$, we conducted linear viscoelastic tests (dynamic tests) with a laboratory rheometer (ARES; Rheometrics) in a parallel plate geometry of the diameter $=$ $8 \mathrm{~mm}$. The test was also made for a sample cast from a $10 \mathrm{wt} \%$ benzene solution and quickly blow-dried at room temperature. This sample mimicked the solventcast film utilized in the photo-isomerization experiments. ${ }^{1}$ The quenched and cast samples exhibited similar viscoelastic relaxation behavior that reflected the large-scale thermal motion of the amorphous cisPPV chains.

\section{RESULTS AND DISCUSSION}

\section{DSC and WAXD for cis-PPV}

Figures 1 and 2, respectively, show the DSC and WAXD profiles of the cis-PPV sample. In Figure 1,

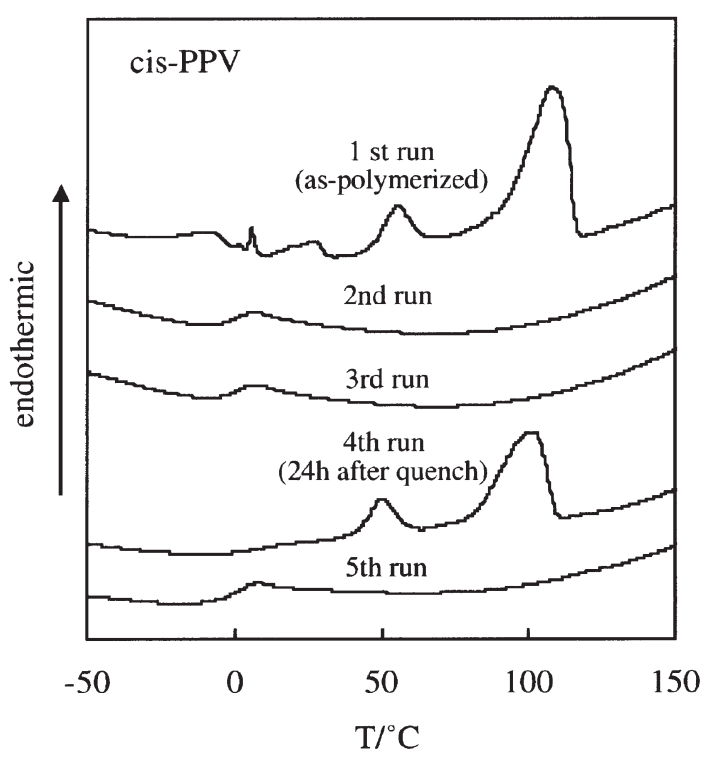

Figure 1. DSC profile of cis-PPV sample obtained under the heating rate of $20^{\circ} \mathrm{C} / \mathrm{min}$.

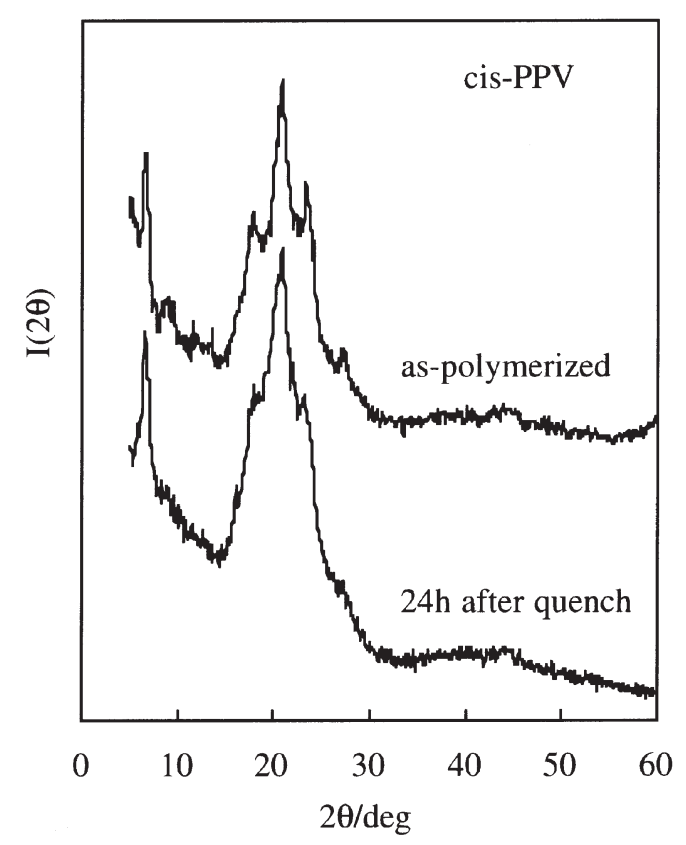

Figure 2. WAXD profiles of as-polymerized and quenched/ annealed cis-PPV samples.

the as-polymerized sample (1st run) exhibits complicated exothermic/endothermic peaks on heating from -10 to $40^{\circ} \mathrm{C}$. This sample was partially crystalline at room temperature, as evidenced from the WAXD peaks seen in Figure 2 (top curve), and the broadness of these peaks indicates that the crystalline is not perfect. From this point of view, the complicated thermal behavior seen at -10 to $40^{\circ} \mathrm{C}$ can be attributed to the glass transition, pre-melting, and recrystallization of the cis-PPV chains in the as-polymerized sample. On further heating, the sample exhibits broad but distinct endothermic peaks at $T \cong 56$ and $109^{\circ} \mathrm{C}$; see the 
first-run curve in Figure 1. These peaks suggest that the as-polymerized sample included two kinds of crystallines having different stabilities: The most stable crystalline exhibiting the higher- $T$ peak may have been either converted from the less stable crystalline exhibiting the lower- $T$ peak (and thus this peak may reflect an isomorphic transition) or already existing in the sample at the beginning of the DSC measurements. In both cases, the melting temperature of the most stable crystalline can be assigned as $T_{\mathrm{m}} \cong$ $109^{\circ} \mathrm{C}$.

In the second-run of the DSC measurement conducted immediately after the first-run, the sample exhibited neither the complicated thermal behavior at -10 to $40^{\circ} \mathrm{C}$ nor the distinct endothermic (melting) behavior at higher $T$; see Figure 1. Instead, the quenched sample just showed the glass transition at $T_{\mathrm{g}} \cong 0{ }^{\circ} \mathrm{C}$, indicating that the sample was supercooled to an amorphous state on quenching. The same behavior was observed in the third-run that was made about $2 \mathrm{~h}$ after the second-run, indicating the lack of recrystallization for few hours after the quench.

After the third-run, the quenched sample was annealed at $25^{\circ} \mathrm{C}$ for $24 \mathrm{~h}$. In the fourth-run (Figure 1), this annealed sample showed no complicated thermal behavior at -10 to $40^{\circ} \mathrm{C}$ but exhibited the endothermic peaks at higher $T$. Thus, the recrystallization occurred after the $24 \mathrm{~h}$ annealing, as confirmed also from the WAXD measurement (bottom curve in Figure 2). However, the endothermic peak temperatures and the WAXD profile of the annealed sample are lower and broader, respectively, than those of the as-polymerized sample, indicating that the recrystallization is not completed by the $24 \mathrm{~h}$ annealing at $25^{\circ} \mathrm{C}$. (In the fifthrun made immediately after the fourth-run, the sample just exhibited the glass transition as it did in the second-run.)

The above results demonstrate that the as-polymerized cis-PPV sample has the partial crystalline structure and the annealed sample has roughly the same structure while the quenched sample remains in the amorphous state for few hours. The crystalline structure and the corresponding thermal behavior are an interesting subject of detailed analysis. However, since the aim of this paper is to examine the mobility of the amorphous cis-PPV chains in relation to their photo-isomerization behavior, we leave this analysis to the future work and hereafter focus on the behavior of the amorphous chains in the quenched sample.

\section{Viscoelastic Behavior of Amorphous cis-PPV Chains}

The as-polymerized cis-PPV sample was charged in the rheometer and allowed to fully melt at $120^{\circ} \mathrm{C}$ $\left(>T_{\mathrm{m}}\right)$. Then, this sample was quenched in the rheometer to several temperatures between 26 and $70^{\circ} \mathrm{C}$

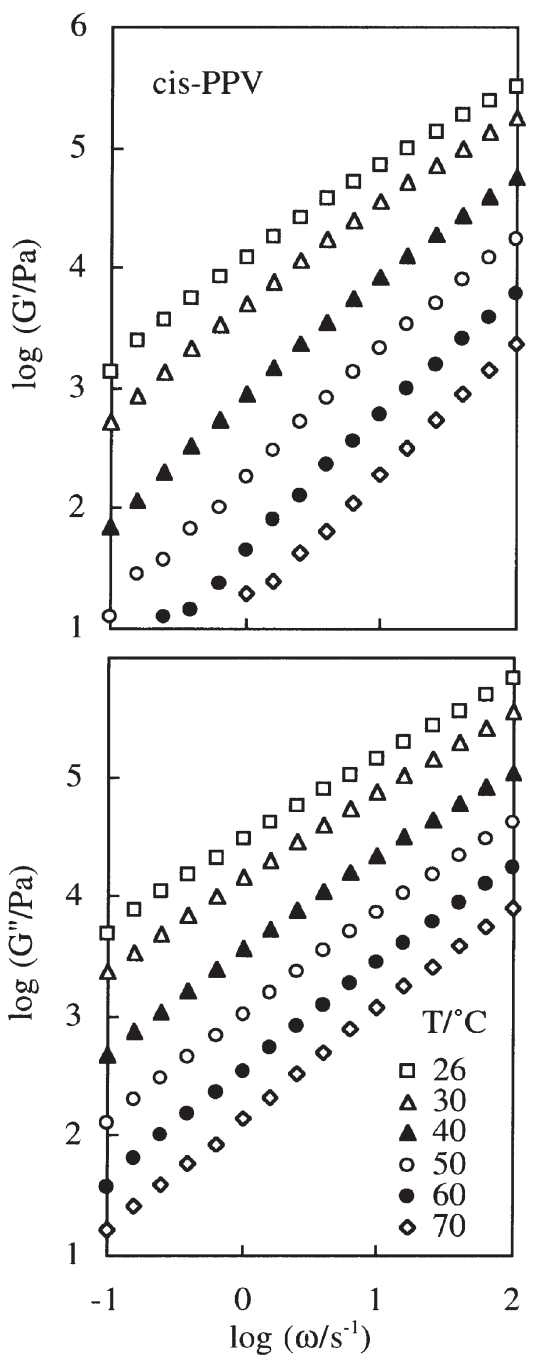

Figure 3. Linear viscoelastic moduli of cis-PPV sample quenched from molten state to several temperatures as indicated.

and the linear viscoelastic, storage and loss moduli $G^{\prime}(\omega)$ and $G^{\prime \prime}(\omega)$ were measured immediately after the quenching as functions of the angular frequency $\omega$. In Figure 3, the $G^{\prime}$ and $G^{\prime \prime}$ data thus obtained are double-logarithmically plotted against $\omega$.

The cis-PPV sample was also cast from a $10 \mathrm{wt} \%$ benzene solution (in a flat glass dish) and quickly blow-dried at room temperature. This sample mimicked the solvent-cast film utilized in the photo-isomerization experiment. ${ }^{1}$ The $G^{\prime}$ and $G^{\prime \prime}$ data at $26^{\circ} \mathrm{C}$ measured for this as-cast/dried sample agreed well with those of the quenched sample (unfilled squares in Figure 3), suggesting that the PPV chains had the same relaxation rate/thermal mobility in the cast and quenched samples.

The $\omega$ dependence of the $G^{\prime}$ and $G^{\prime \prime}$ data seen in Figure 3 is similar to the Rouse-like dependence observed for a wide variety of low- $M$, non-crystallizable (intrinsically amorphous) homopolymers at $T>T_{\mathrm{g}}{ }^{5,6}$ Since these low- $M$ homopolymer liquids obey the time-temperature superposition, ${ }^{5}$ we pursued the sim- 


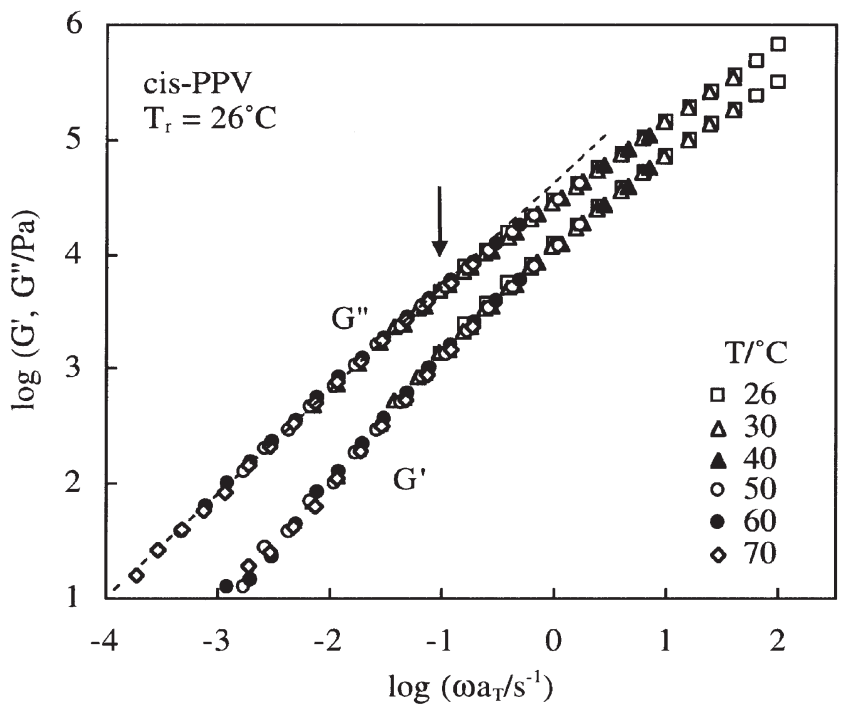

Figure 4. Master curves of linear viscoelastic moduli of cisPPV sample quenched from molten state to several temperatures as indicated.

ilarity by examining the validity of this superposition for the cis-PPV quenched sample. For a reference temperature of $T_{\mathrm{r}}=299 \mathrm{~K}\left(=26^{\circ} \mathrm{C}\right)$, the superposed master curves of the $G^{\prime}$ and $G^{\prime \prime}$ data are shown in Figure 4, and the shift factor $a_{\mathrm{T}}$ utilized in this superposition is plotted against temperature in Figure 5 (squares). The superposition excellently works (Figure 4), and the $a_{\mathrm{T}}$ data are well described by a WLF equation ${ }^{5}$ shown with the solid curve in Figure 5:

$$
\begin{aligned}
& \log a_{\mathrm{T}}=-\frac{C_{1}\left(T-T_{\mathrm{r}}\right)}{C_{2}+T-T_{\mathrm{r}}} \quad \text { with } C_{1}=7.576, \\
& C_{2}=351.0 \mathrm{~K}, \text { and } T_{\mathrm{r}}=299 \mathrm{~K}
\end{aligned}
$$

The $a_{\mathrm{T}}$ data are equally well described by the so-called universal WLF equation (known to be valid for various homopolymers ${ }^{5}$ ) shown with the small plus symbols in Figure 5:

$$
\begin{aligned}
\log a_{\mathrm{T}}= & -\frac{8.86\left\{T-\left(T_{\mathrm{g}}^{\mathrm{o}}+50\right)\right\}}{101.6+T-\left(T_{\mathrm{g}}^{\mathrm{o}}+50\right)} \\
& +\frac{8.86\left\{T_{\mathrm{r}}-\left(T_{\mathrm{g}}^{\mathrm{o}}+50\right)\right\}}{101.6+T_{\mathrm{r}}-\left(T_{\mathrm{g}}^{\mathrm{o}}+50\right)}
\end{aligned}
$$

Here, $a_{\mathrm{T}}$ is the shift factor for the chosen $T_{\mathrm{r}}\left(=26^{\circ} \mathrm{C}\right)$, and $T_{\mathrm{g}}^{\mathrm{o}}$ is the glass transition temperature measured in the static condition (i.e., measured with DSC at a vanishingly small heating rate.) In Figure $5, T_{\mathrm{g}}^{\mathrm{o}}$ was chosen to be $-22^{\circ} \mathrm{C}$. This $T_{\mathrm{g}}^{\mathrm{o}}$ value seems to be consistent with the $T_{\mathrm{g}}$ value ( $\cong 0^{\circ} \mathrm{C}$ ) obtained at the heating rate of $20 \mathrm{deg} / \mathrm{min}$.

All above results indicate that the cis-PPV chains in the quenched/cast samples exhibit the viscoelastic relaxation indistinguishable from that of the intrinsically amorphous homopolymer liquids. These results, dem-

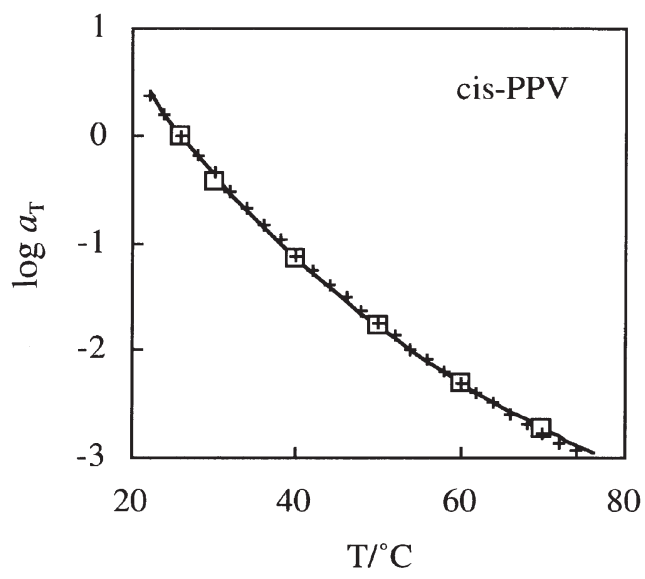

Figure 5. Temperature dependence of the shift factor for the master curves shown in Figure 4. The solid curve and small plus symbols, respectively, indicate the WLF equations (eqs 1 and 2).

onstrating the considerable stableness of the supercooled amorphous state of the cis-PPV chains, are consistent with those obtained from the DSC measurements.

\section{Rate of Conformational Change of Amorphous cis- PPV Chains}

Because the cis-PPV chains in the quenched/cast samples behave as the usual amorphous chains, we can estimate the time required for the thermal change of large-scale conformation (orientational relaxation of the whole contour of the chain ${ }^{6}$ ) as the viscoelastic relaxation time. If the low- $\omega$ tails of the $G^{\prime}$ and $G^{\prime \prime}$ data characterized with the power-law dependencies, ${ }^{5}$ $G^{\prime} \propto \omega^{2}$ and $G^{\prime \prime} \propto \omega$, are observed, the terminal relaxation time is unequivocally evaluated as ${ }^{7}\langle\tau\rangle_{\mathrm{w}}=$ $\left[G^{\prime} / \omega^{2}\right]_{\omega \rightarrow 0} /\left[G^{\prime \prime} / \omega\right]_{\omega \rightarrow 0}$. However, for the cis-PPV chains being synthesized via polycondensation and having a rather broad molecular weight distribution ${ }^{1}$ $\left(M_{\mathrm{w}} / M_{\mathrm{n}}=2.16\right)$, the relaxation mode distribution is broad and the low- $\omega$ tail of $G^{\prime}$ sensitive to the weak but slow modes is not observed even at the lowest $\omega$ and highest $T$ examined; see Figure 4. Thus, $\langle\tau\rangle_{\mathrm{w}}$ cannot be evaluated for those chains. For this reason, we made a rough estimate for the terminal relaxation time in the following way.

Since the $G^{\prime \prime}$ data are insensitive to the weak and slow relaxation modes, their low- $\omega$ tail (satisfying the proportionality $G^{\prime \prime} \propto \omega$ ) is observed within the scatter of the data points, as shown with the dotted line in Figure 4. The $G^{\prime \prime}$ data deviate downward from this tail with increasing $\omega$, and the terminal relaxation time $\langle\tau\rangle$ can be roughly estimated from the frequency for the onset of this deviation, $\omega_{\mathrm{d}}$ (shown with the arrow), as

$$
\langle\tau\rangle \cong 1 / \omega_{\mathrm{d}} \cong 10 \mathrm{~s} \text { at } 26^{\circ} \mathrm{C}
$$

Although this $\langle\tau\rangle$ is a rough estimate, we can still con- 
clude that the time $\langle\tau\rangle^{\prime}(\cong\langle\tau\rangle)$ necessary for the thermal changes in the large-scale conformation of the amorphous cis-PPV chains is of the order of $10 \mathrm{~s}$ at $26^{\circ} \mathrm{C}$.

Here, we should note the important interplay between a given chain (probe chain) undergoing the cis-to-trans photo-isomerization and the chains surrounding this probe. Since this isomerization should result in a sweeping motion of the probe against the surrounding cis-PPV chains, the isomerization would efficiently proceed only when the surrounding chains exhibit quick thermal change of their conformation and make a room for the sweeping motion. Thus, the time for the thermal change of the conformation, $\langle\tau\rangle^{\prime}$, should be much shorter than the time for the completion of the photo-isomerization, $t_{\text {iso }}$. Indeed, this is the case for the cis-PPV chains; $\langle\tau\rangle^{\prime}=O(10) \mathrm{s}$ and $t_{\text {iso }} \cong 45 \mathrm{~min}=O\left(10^{3}\right) \mathrm{s}^{1}$

In relation to this large difference between $\langle\tau\rangle^{\prime}$ and $t_{\text {iso, }}$, we should note that the trans-PPV chains are formed as the photo-isomerization proceeds and thus the isomerization slows down if the thermal mobility of these trans-PPV chains is smaller than the mobility of the cis-PPV chains. This point was examined from the DSC measurement for the all-trans PPV sample recovered from a UV-irradiated dilute solution of cis-PPV chains in benzene. (At room temperature, the recovered sample was glassy and the characteristic time for the large-scale motion of the trans-PPV chain could not be determined viscoelastically.)

Figure 6 shows the DSC profile of the trans-PPV sample in the fully dried state. In the range of temperature examined $\left(\leq 150^{\circ} \mathrm{C}\right)$, the sample exhibits no melting peak of crystallines but shows the glass transition at $T_{\mathrm{g}} \cong 50^{\circ} \mathrm{C}$ under the heating rate of $20^{\circ} \mathrm{C} /$ min. This $T_{\mathrm{g}}$ is significantly higher than $T_{\mathrm{g}}$ of cis-PPV $\left(\cong 0{ }^{\circ} \mathrm{C}\right)$ obtained under the same heating rate, and the trans-PPV chains in the bulk sample are glassy at room temperature unless they are plasticized with the cis-PPV chains. This result suggests that the average thermal mobility of the chains surrounding a probe cis-PPV chain decreases as the photo-isomerization proceeds and the content of the trans-PPV

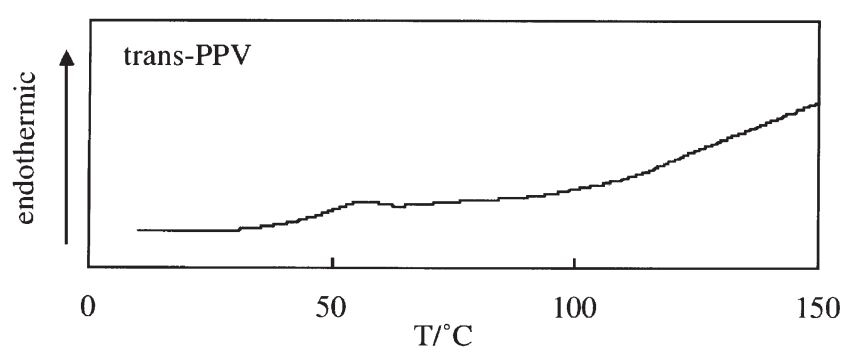

Figure 6. DSC profile of trans-PPV sample recovered from a UV-irradiated cis-PPV/benzene solution. chain increases accordingly. This decrease of the mobility should result in the slowing down of the isomerization. The large difference between $\langle\tau\rangle^{\prime}$ and $t_{\text {iso }}$ seems to reflect this slowing down.

\section{CONCLUDING REMARKS}

In order to examine the thermal mobility of cis-PPV chains in relation to their photo-isomerization into the trans form, we have conducted the thermal, structural, and viscoelastic tests for cis-PPV samples quenched from the molten state and/or cast from a solution. The amorphous cis-PPV chains in these samples had a low $T_{\mathrm{g}}\left(\cong 0{ }^{\circ} \mathrm{C}\right)$ and were in the liquid state at room temperature. The time required for the thermal change of the large scale conformation of these chains, $\langle\tau\rangle^{\prime}=$ $O(10)$ s at room temperature, was significantly shorter than the time for completion of the photo-isomerization, $t_{\text {iso }} \cong 45 \mathrm{~min}=O\left(10^{3}\right) \mathrm{s}$. The large difference between these $\langle\tau\rangle^{\prime}$ and $t_{\text {iso }}$ values are consistent with the efficient isomerization observed for the cis-PPV cast film.

Acknowledgment. The authors thank Dr. H. Masai and Ms. M. Mizuno at Professor T. Yoko's lab, ICR, Kyoto University, for their help for DSC measurements and Mr. T. Konishi at Professor T. Kanaya's lab, ICR, Kyoto University, for his help for WAXD measurements.

\section{REFERENCES}

1. H. Katayama, M. Nagao, T. Nishimura, Y. Matsui, K. Umeda, K. Akamatsu, T. Tsuruoka, H. Nawafune, and F. Ozawa, J. Am. Chem. Soc., 127, 4350 (2005).

2. H. Katayama, M. Nagao, R. Moriguchi, and F. Ozawa, J. Organomet. Chem., 676, 49 (2003).

3. a) J. H. Burroughes, D. D. C. Bradley, A. R. Brown, R. N. Marks, K. Mackay, R. H. Friend, P. L. Burn, and A. B. Holmes, Nature, 347, 539 (1990).

b) P. L. Burn, A. B. Holmes, A. Kraft, D. D. C. Bradley, A. R. Brown, R. H. Friend, and R. W. Gymer, Nature, 356, 47 (1992).

c) G. Gustafsson, Y. Cao, G. M. Treacy, F. Klavetter, N. Colaneri, and A. J. Heeger, Nature, 357, 477 (1992).

d) N. C. Greenham, S. C. Moratti, D. D. C. Bradley, R. H. Friend, P. L. Burn, and A. B. Holmes, Nature, 365, 628 (1993).

e) R. J. O. M. Hoofman, M. P. de Haas, L. D. A. Siebbeles, and J. M. Warman, Nature, 392, 54 (1998).

f) S. Welter, K. Brunner, J. W. Hofstraat, and L. De Cola, Nature, 421, 54 (2003).

4. a) N. S. Sariciftci, D. Braun, C. Zhang, V. I. Srdanov, A. J. Heeger, G. Stucky, and F. Wudl, Appl. Phys. Lett., 62, 585 (1993).

b) J. J. M. Halls, K. Pichler, R. H. Friend, S. C. Moratti, and A. B. Holmes, Appl. Phys. Lett., 68, 3120 (1996). 
c) H. Ago, K. Petritsch, M. S. P. Shaffer, A. H.Windle, and R. H. Friend, Adv. Mater., 11, 1281 (1999).

d) A. J. Breeze, Z. Schlesinger, S. A. Carter, and P. J. Brock, Phys. Rev. B, 64, 125205 (2001).

e) D. Gebeyehu, C. J. Brabec, F. Padinger, T. Fromherz, J. C. Hummelen, D. Badt, H. Schindler, and N. S. Sariciftci, Synth. Met., 118, 1 (2001).

f) J. K. J. van Duren, X. N. Yang, J. Loos, C. W. T. Bulle-Lieuwma, A. B. Sieval, J. C. Hummelen, and R. A. J.
Janssen, Adv. Funct. Mater., 14, 425 (2004).

g) H. Hoppe, M. Niggemann, C. Winder, J. Kraut, R. Hiesgen, A. Hinsch, D. Meissner, and N. S. Sariciftci, Adv. Funct. Mater., 14, 1005 (2004).

5. J. D. Ferry, "Viscoelastic Properties of Polymers," 3rd ed., Wiley, New York, 1980.

6. H. Watanabe, Prog. Polym. Sci., 24, 1253 (1999).

7. W. W. Graessley, Adv. Polym. Sci., 16, 1 (1974). 